\title{
GENERALIZED STUMMEL CLASS AND MORREY SPACES OF NONHOMOGENEOUS TYPE
}

\author{
Wono Setya Budhi ${ }^{1}$, Idha Sihwaningrum ${ }^{2}$, And Yudi Soeharyadi ${ }^{3}$ \\ ${ }^{1}$ Bandung Institute of Technology, Indonesia, wono@math.itb.ac.id \\ ${ }^{2}$ Jenderal Soedirman University, Indonesia, idhasihwaningrum@yahoo.com \\ ${ }^{3}$ Bandung Institute of Technology, Indonesia, yudish@math.itb.ac.id
}

\begin{abstract}
In the context of the spaces of nonhomogeneous type, in this paper we study a relation between the generalized Stummel class and the generalized Morrey spaces. The stummel class is a class of functions related to local behavior of mapping by fractional integral operators. Meanwhile, the generalized Morrey spaces are classes of functions related to local behavior of Hardy-Littlewood maximal function. Our results employ the doubling condition of functions under consideration.

Key words: Generalized Stummel class, generalized Morrey spaces, spaces of nonhomogeneous type.
\end{abstract}

\begin{abstract}
Abstrak. Dalam konteks ruang bertipe tak homogen, pada makalah ini dibahas hubungan antara kelas Stummel yang diperumum dan ruang Morrey yang diperumum. Kelas Stummel yang diperumum adalah kelas fungsi yang berkaitan dengan sifat lokal pemetaan oleh operator integral, sedangkan ruang Morrey yang diperumum adalah kelas fungsi yang berkaitan dengan sifat lokal dari fungsi maksimal Hardy-Littlewood. Hasil pada makalah ini diperoleh dengan memanfaatkan kondisi doubling dari fungsi-fungsi yang digunakan.
\end{abstract}

Kata kunci: Kelas Stummel yang diperumum, ruang Morrey yang diperumum, ruang bertipe tak homogen.

\section{INTRODUCTION}

Let $B(x, r)$ be a ball centered at $x \in \mathbb{R}^{d}$ with radius $r>0$; and for $k>0$, let us set $B(x, k r)$ as the ball concentric to $B(x, r)$ with radius $k r$. The Euclidean space $\mathbb{R}^{d}$, endowed with a positive Radon measure $\mu$, is a homogeneous space if $\mu$ satisfies the doubling condition, that is there exists a constant $C>0$ such that for every

2000 Mathematics Subject Classification: 46E30, 43A15.

Received: 19-08-2013, revised: 27-05-2014, accepted: 16-06-2014. 
ball $B(x, r)$ we have

$$
\mu(B(x, 2 r)) \leq C \mu(B(x, r)) .
$$

We refer to [1] for a pioneer work on homogeneous spaces. The space $\mathbb{R}^{d}$ equipped with the usual Lebesgue measure is an example of homogeneous space.

Since 1970's, the doubling condition has been an important assumption in developing some theories in harmonic analysis. However, some recent results, see $[4,7,8,12,13]$, show that doubling condition assumption is not as significant as thought before, that is some results can be obtained without doubling condition. The space $\left(\mathbb{R}^{d}, \mu\right)$ where the Radon measure $\mu$ does not satisfy the doubling condition is known as the nonhomogeneous space. In this paper, we will particularly work with $\mu$ which satisfies the growth condition:

$$
\mu(B(x, r)) \leq C^{*} r^{n}
$$

for every ball $B(x, r)$. In the growth condition (1), $n$ is a fixed real number such that $0<n \leq d$ and the constant $C^{*}$ is independent of $x$ and $r$. Without loss of generality, we will assume that $C^{*} \geq 1$.

In the spaces of homogeneous type, Eridani and Gunawan [2] introduced the generalized Stummel class $S_{\psi}:=S_{\psi}\left(\mathbb{R}^{d}\right)$, for $\psi:(0, \infty) \rightarrow(0, \infty)$, which is defined by

$$
S_{\psi}:=\left\{f \in L_{\mathrm{loc}}^{1}(\mu): \lim _{r \rightarrow 0} \zeta_{\psi} f(r)=0\right\} .
$$

Here,

$$
\zeta_{\psi} f(r):=\sup _{x \in \mathbb{R}^{d}} \int_{|x-y|<r}|f(y)| \frac{\psi(|x-y|)}{|x-y|^{n}} d y
$$

is called the Stummel modulus of $f$. The class $S_{\psi}$ is a generalization of the Stummel class $S_{p}:=S_{p}\left(\mathbb{R}^{d}\right)($ for $1<p<d)$ where

$$
S_{p}:=\left\{f \in L_{\mathrm{loc}}^{1}(\mu): \lim _{r \rightarrow 0} \sup _{x \in \mathbb{R}^{d}} \int_{|x-y|<r} \frac{|f(y)|)}{|x-y|^{d-p}} d y=0\right\} .
$$

This class was introduced by Ragusa and Zamboni [9]. If $\psi(t)=t^{p}$ and $1<$ $p<d$, where $d$ is the dimension of the Euclidean space $\mathbb{R}^{d}$, then we have $S_{\psi}=$ $S_{p}$; meanwhile if $\psi(t)=t^{2}$, the class $S_{\psi}$ is reduced to the classical StummelKato class. The relevance of the classical Stummel-Kato class in the study of Schrödinger operators can be found for example in [14]. For homogeneous type spaces, Ragusa and Zamboni [9] verified a relation between the Morrey spaces and the Stummel class $S_{p}$; meanwhile Eridani and Gunawan [2] as well as Gunawan et al. [6] established relations between the generalized Morrey spaces and the generalized Stummel class $S_{\psi}$ (of homogeneous type spaces). The Stummel class is a class of functions related to local behavior of mapping by generalized fractional integral operators; and the generalized Morrey spaces are classes of functions related to local behavior of Hardy-Littlewood maximal function. We may see [5, 10] for recent results in fractional integral operators and maximal operators. In this paper, we extend the results of [2] and [6] in nonhomogeneous setting. 


\section{Main Results}

For $\psi:(0, \infty) \rightarrow(0, \infty)$, we define the generalized Stummel class $S_{\psi}(\mu):=$ $S_{\psi}\left(\mathbb{R}^{d}, \mu\right)$ of nonhomogeneous type by

$$
S_{\psi}(\mu):=\left\{f \in L_{\mathrm{loc}}^{1}(\mu): \lim _{r \rightarrow 0} \eta_{\psi} f(r)=0\right\} .
$$

Here, the nondecreasing function

$$
\eta_{\psi} f(r):=\sup _{x \in \mathbb{R}^{d}} \int_{|x-y|<r}|f(y)| \frac{\psi(|x-y|)}{|x-y|^{n}} d \mu(y), \quad 0<n \leq d,
$$

is the Stummel modulus of $f$.

Now, for $\phi:(0, \infty) \rightarrow(0, \infty)$ and $k>1$, we define the generalized Morrey space of nonhomogeneous type $\mathcal{M}^{1, \phi}(k, \mu)=\mathcal{M}^{1, \phi}\left(\mathbb{R}^{d}, k, \mu\right)$ to be the space of all functions $f \in L_{\text {loc }}^{1}(\mu)$ for which

$$
\left\|f: \mathcal{M}^{1, \phi}(k, \mu)\right\|:=\sup _{B(x, r)} \frac{1}{\phi(\mu(B(x, k r)))} \frac{1}{\mu(B(x, k r))} \int_{B(x, r)}|f(y)| d \mu(y)<\infty .
$$

We may refer [3] for definition of the analog spaces in the homogeneous setting.

Throughout this paper, for $t>0$, we will always assume that $\phi(t)$ is an almost decreasing function and $t \phi(t)$ is an almost increasing function. This also means that we always assume the doubling condition on $\phi$. The function $\phi$ is doubling if there exists a constant $C>0$ such that

$$
\frac{1}{C} \leq \frac{\phi(s)}{\phi(t)} \leq C \quad \text { whenever } \quad 1 \leq \frac{s}{t} \leq 2
$$

Unless otherwisely stated, for the rest of the paper, we denote by $C$ a positive constant which may vary from line to line. Recall that $\phi$ is an almost decreasing [increasing] function if there exists $C$ such that $\phi(s) \geq C \phi(t)[\phi(s) \leq C \phi(t)]$ whenever $s<t$. Moreover, our generalized Morrey spaces are independent of $k$ since for $k_{1}>k_{2}>1$ we have

$$
C_{1}\left\|f: \mathcal{M}^{1, \phi}\left(k_{1}, \mu\right)\right\| \leq\left\|f: \mathcal{M}^{1, \phi}\left(k_{2}, \mu\right)\right\| \leq C_{2}\left\|f: \mathcal{M}^{1, \phi}\left(k_{1}, \mu\right)\right\|
$$

where $C_{1}, C_{2}>0$.

Our first result states the inclusion of the generalized Morrey spaces into the Stummel class.

Theorem 2.1. Assume that $t^{-n} \psi(t)$ is almost decreasing. If

$$
\int_{0}^{1} \frac{\psi(t) \phi\left(t^{n}\right)}{t} d t<\infty
$$

then $\mathcal{M}^{1, \phi}(k, \mu) \subseteq S_{\psi}(\mu)$.

Proof. Let $f \in \mathcal{M}^{1, \phi}(2, \mu)$. (This suffices as our generalized Morrey spaces $\mathcal{M}^{1, \phi}(k, \mu)$ are independent of $k$.) By using the almost decreasing of $t^{-n} \psi(t)$ and 
the doubling condition of $\phi$, we have

$$
\begin{aligned}
& \int_{2^{j} r \leq|x-y|<2^{j+1} r} \frac{|f(y)| \psi(|x-y|)}{|x-y|^{n}} d \mu(y) \\
& \leq C \frac{\psi\left(2^{j} r\right)}{\left(2^{j} r\right)^{n}} \int_{2^{j} r \leq|x-y|<2^{j+1} r}|f(y)| d \mu(y) \\
& \leq C \frac{\psi\left(2^{j} r\right)}{\left(2^{j} r\right)^{n}} \phi\left(\mu\left(B\left(x, 2^{j+2} r\right)\right)\right) \mu\left(B\left(x, 2^{j+2} r\right)\right)\left\|f: \mathcal{M}^{1, \phi}(2, \mu)\right\| \\
& \leq C \frac{\psi\left(2^{j} r\right)}{\left(2^{j} r\right)^{n}} \phi\left(C^{*}\left(2^{j+2} r\right)^{n}\right)\left(2^{j+2} r\right)^{n}\left\|f: \mathcal{M}^{1, \phi}(2, \mu)\right\| \\
& \leq C \int_{2^{j-1} r}^{2^{j} r} \frac{\psi(t)}{t^{n}} \phi\left(t^{n}\right) t^{n} \frac{d t}{t}\left\|f: \mathcal{M}^{1, \phi}(2, \mu)\right\| .
\end{aligned}
$$

Then

$$
\begin{gathered}
\int_{|x-y|<r} \frac{|f(y)| \psi(|x-y|)}{|x-y|^{n}} d \mu(y) \\
\quad=\sum_{j=-\infty}^{-1} \int_{2^{j} r \leq|x-y|<2^{j+1} r} \frac{|f(y)| \psi(|x-y|)}{|x-y|^{n}} d \mu(y) \\
\leq C \sum_{j=-\infty}^{-1} \int_{2^{j-1} r}^{2^{j} r} \frac{\psi(t)}{t^{n}} \phi\left(t^{n}\right) t^{n} \frac{d t}{t}\left\|f: \mathcal{M}^{1, \phi}(2, \mu)\right\| \\
=C\left\|f: \mathcal{M}^{1, \phi}(2, \mu)\right\| \int_{0}^{r / 2} \frac{\psi(t) \phi\left(t^{n}\right)}{t} d t
\end{gathered}
$$

Since $\int_{0}^{1} \frac{\psi(t) \phi\left(t^{n}\right)}{t} d t<\infty$, we obtain $\int_{0}^{r / 2} \frac{\psi(t) \phi\left(t^{n}\right)}{t} d t \rightarrow 0$ whenever $r \rightarrow 0$. This proves our assertion

Our next result is the inclusion of the Stummel class into the generalized Morrey spaces. We will present it in the following theorem.

Theorem 2.2. Assume that $t^{-n} \psi(t)$ is almost decreasing. If $f \in S_{\psi}(\mu)$ and there exists a positif constant $C$ such that

$$
\int_{0}^{r} \frac{t^{n-1} \eta_{\psi} f(t)}{\psi(t)} d t \leq C \mu(B(x, r)) \phi(\mu(B(x, r)))
$$

for all ball $B(x, r)$, then $f \in \mathcal{M}^{1, \phi}(k, \mu)$. 
Proof. Given ball $B(x, r)$ where $x \in \mathbb{R}^{d}$ and $r>0$ and for $f \in S_{\psi}(\mu)$, we have

$$
\begin{aligned}
\int_{|x-y|<r}|f(y)| d \mu(y) & =\sum_{j=-\infty}^{-1} \int_{2^{j} r \leq|x-y|<2^{j+1} r}|f(y)| d \mu(y) \\
& \leq C \sum_{j=-\infty}^{-1} \frac{\left(2^{j+1} r\right)^{n}}{\psi\left(2^{j+1} r\right)} \int_{|x-y|<2^{j+1} r} \frac{|f(y)| \psi(|x-y|)}{|x-y|^{n}} d \mu(y) \\
& \leq C \sum_{j=-\infty}^{-1} \frac{\left(2^{j+1} r\right)^{n} \eta_{\psi} f\left(2^{j+1} r\right)}{\psi\left(2^{j+1} r\right)} \\
& =C \sum_{j=-\infty}^{0} \frac{\left(2^{j} r\right)^{n} \eta_{\psi} f\left(2^{j} r\right)}{\psi\left(2^{j} r\right)} .
\end{aligned}
$$

Now, we apply the almost decreasing of $t^{-n} \psi(t)$ to get

$$
\begin{aligned}
\int_{|x-y|<r}|f(y)| d \mu(y) & \leq C \sum_{j=-\infty}^{0} \frac{\left(2^{j} r\right)^{n} \eta_{\psi} f\left(2^{j} r\right)}{\psi\left(2^{j} r\right)} \\
& \leq C \sum_{j=-\infty}^{0} \int_{2^{j} r}^{2^{j+1} r} \frac{t^{n-1} \eta_{\psi} f(t)}{\psi(t)} d t \\
& =C \int_{0}^{2 r} \frac{t^{n-1} \eta_{\psi} f(t)}{\psi(t)} d t \\
& \leq C \mu(B(x, 2 r) \phi(\mu(B(x, 2 r)) .
\end{aligned}
$$

The last inequality lead us to $f \in \mathcal{M}^{1, \phi}(2, \mu)$. Therefore, the desired result follows.

\section{Further Results}

For $\psi: \mathbb{R}^{d} \times(0, \infty) \rightarrow(0, \infty)$, define $S_{\psi}(\mu)$ by the same way in Section 2 with

$$
\eta_{\psi} f(r):=\sup _{x \in \mathbb{R}^{d}} \int_{|x-y|<r}|f(y)| \frac{\psi(x,|x-y|)}{|x-y|^{n}} d \mu(y),
$$

see also Section 4 in [6]. For $\phi: \mathbb{R}^{d} \times(0, \infty) \rightarrow(0, \infty)$, let $\mathcal{M}^{1, \phi}(k, \mu)$ be the space of all functions $f$ such that

$$
\left\|f: \mathcal{M}^{1, \phi}(k, \mu)\right\|:=\sup _{B(x, r)} \frac{1}{\phi(x, \mu(B(x, k r)))} \frac{1}{\mu(B(x, k r))} \int_{B(x, r)}|f(y)| d \mu(y)<\infty .
$$

We always assume that there exists a positive constant $C$ such that

$$
\phi(x, t) \leq C \phi(x, s), \quad s \phi(x, s) \leq C t \phi(x, t)
$$

whenever $x \in \mathbb{R}^{d}, 0<s \leq t<\infty$. Then, we can prove the following by the same way as Theorems 2.1 and 2.2 : 
Theorem 3.1. Assume that there exists a positive constant $C$ such that

$$
\begin{gathered}
t^{-n} \psi(x, t) \leq C s^{-n} \psi(x, s) \\
\text { whenever } x \in \mathbb{R}^{d}, 0<s \leq t<\infty \text {. If } \\
\lim _{r \rightarrow 0} \sup _{x \in \mathbb{R}^{d}} \int_{0}^{r} \frac{\psi(x, t) \phi\left(x, t^{n}\right)}{t} d t=0,
\end{gathered}
$$

then $\mathcal{M}^{1, \phi}(k, \mu) \subseteq S_{\psi}(\mu)$. Conversely, if $f \in S_{\psi}(\mu)$ and there exists a positif constant $C$ such that

$$
\int_{0}^{r} \frac{t^{n-1} \eta_{\psi} f(t)}{\psi(x, t)} d t \leq C \mu(B(x, r)) \phi(x, \mu(B(x, r)))
$$

for all ball $B(x, r)$, then $f \in \mathcal{M}^{1, \phi}(k, \mu)$.

Acknowledgement The research was supported by ITB Research Grant 2009, No. 277/K01.7/ PL/2009; and this paper was completed during a visit to Department of Mathematics Kyoto University which was supported by GCOE Program 2011 of Kyoto University. We would like to thank H. Gunawan and Y. Sawano for many fruitful discussions. Special thank also go to the anonymous referee for improving our paper, especially for adding Section 3.

\section{REFERENCES}

[1] Coifman, R.R. and de Gusmán, M., "Singular Integrals and Multiplier on Homogeneous Spaces", Rev. Un. Mat. Argentina, 25 (1970/1971) 137-143.

[2] Eridani and Gunawan, H., "Stummel Class and Morrey Spaces", Southeast Asian Bull. Math., 29 (2005), 1053-1056.

[3] Eridani, Gunawan,H., and Nakai, E., "On the Generalized Fractional Integral Operators", Sci. Math. Jpn., 60 (2004), 539-550.

[4] García-Cuerva, J. and Gatto, A.E., "Boundedness Properties of Fractional Integral Operators Associated to Non-doubling Measures", Studia Math., 162 (2004), 245-261.

[5] García-Cuerva, J. and Martell,J.M., "Two Weight Norm Inequalities for Maximal Operators and Fractional Integrals on Non-homogeneous Spaces", Indiana Univ. Math. J., 50 (2001), $1241-1280$.

[6] Gunawan, H., Nakai, E., Sawano, Y., and Tanaka, H., "Generalized Stummel Class and Morrey Spaces", Publications de l'Institut Mathematique, 92 (2012), 127-138. (Open Access DOI: 10.2298/PIM1206127G)

[7] Gunawan, H., Sawano, Y., and Sihwaningrum, I., "Fractional Integral Operators in Nonhomogeneous Spaces", Bull. Aust. Math. Soc., 80: 2 (2009), 324-334.

[8] Nazarov, F., Treil, S., and Volberg, A., "Weak Type Estimates and Cotlar Inequalities for Caldern-Zygmund Operators on Nonhomogeneous Space", Internat. Math. Res. Notices, 9 (1998), 463-487.

[9] Ragusa, M.A. and Zamboni, P., "A Potential Theoritic Inequality", Czech. Math. J., 51 (2001), 55-65.

[10] Sawano,Y., "Sharp Estimates of the Modified Hardy-Littlewood Maximal Operator on the Nonhomogeneous Space via Covering Lemmas", Hokkaido Math. J. 34 (2005), 435-458. 
[11] Sawano, Y. and Tanaka, H., "Morrey Spaces for Non-doubling Measures", Acta Math. Sinica, 1 (2006), 153-172.

[12] Sihwaningrum, I., Suryawan, H.P., and Gunawan, H., "Fractional Integral Operators and Olsen Inequality on Non-homogeneous Spaces", Aust. J. Math. Anal. Appl., 7 (2010), Issue 1, Article 14, 1-6.

[13] Tolsa, X., "BMO, $H^{1}$, and Caldern-Zygmund Operators for Non Doubling Measures", Math. Ann., 319 (2001), 89-149.

[14] Zheng, S., "Time Decay for Schrödinger Equation with Rough Potentials", Analysis in Theory and Applications, 23 (2007), 375-379. 